\title{
Bromine Precursor Mediated Synthesis of Shape Controlled Cesium Bromide Nano-platelets and Their Mechanism Study by DFT Calculation
}

Jae Young Noh, ${ }^{\ddagger a}$ Seungmin Baek, ${ }^{\text {a }}$ Ju Hyun Park, ${ }^{\text {b }}$ Sang Kyu Kwak, ${ }^{* b}$ Sang-Wook Kim*a

a Department of Molecular Science and Technology, Ajou University, Suwon 16499, Republic of Korea

${ }^{b}$ Department of Energy Engineering, School of Energy and Chemical Engineering, Ulsan National Institute of Science and Technology (UNIST), Ulsan 44919, Republic of Korea

(a)

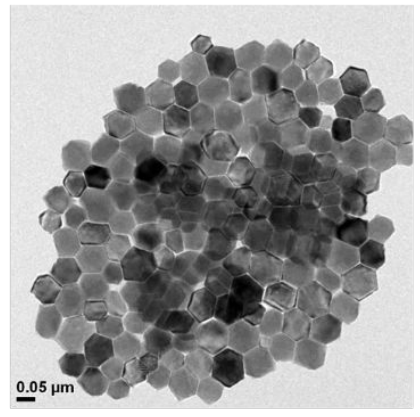

(c)

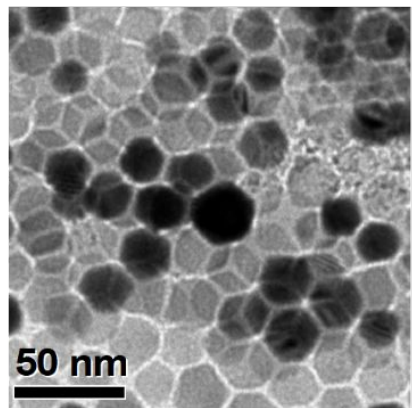

(b)

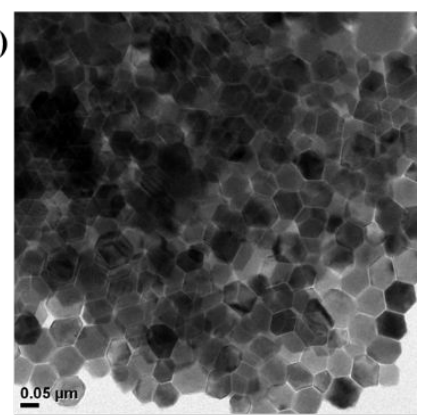

(d)

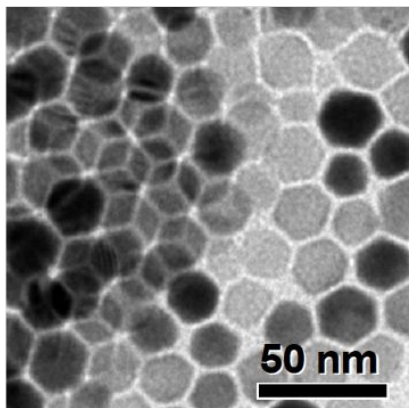

Figure S1. TEM image of Stacked CsBr plates synthesized using HBr.(a-d) Since the thickness of CsBr particles is very thin, its lattice can be clearly shown underlying particle among the overlapped $\mathrm{CsBr}$, and which proves that $\mathrm{CsBr}$ exists as a plate. 
Table S1. Interplanar distance according to the miller indices of the possible growth surfaces of CsBr.

\begin{tabular}{ccc}
\hline h & k 1 & $\mathrm{~d}_{\mathrm{hkl}}(\AA)$ \\
\hline$\left\{\begin{array}{lll}1 & 0 & 0\end{array}\right\}$ & 4.29 \\
$\left\{\begin{array}{lll}1 & 1 & 0\end{array}\right\}$ & 3.03 \\
$\left\{\begin{array}{lll}1 & 1 & 1\end{array}\right\}$ & 2.48 \\
$\left\{\begin{array}{lll}2 & 1 & 0\end{array}\right.$ & 1.92 \\
$\left\{\begin{array}{llll}2 & 1 & 1\end{array}\right\}$ & 1.75 \\
\hline
\end{tabular}

Table S2. Lattice parameters $a$ and $b$ and number density of dangling Cs $(\theta)$ on each surface systems. For the polar surface, $\theta$ was calculated assuming equal to (the number of dangling Cs/area) in Cs-terminated surface. All angles of the surface systems are $90^{\circ}$.

\begin{tabular}{cccc}
\hline $\mathrm{h} \mathrm{k} 1$ & $\mathrm{a}(\AA)$ & $\mathrm{b}(\AA)$ & $\theta\left(\AA^{-2}\right)$ \\
\hline$\left\{\begin{array}{lll}1 & 0 & 0\end{array}\right.$ & 12.8748 & 12.8748 & 0.0543 \\
$\left\{\begin{array}{lll}1 & 1 & 0\end{array}\right.$ & 12.8748 & 12.1385 & 0.0384 \\
$\left\{\begin{array}{lll}1 & 1 & 1\end{array}\right\}$ & 10.5122 & 12.1385 & 0.0627 \\
$\left\{\begin{array}{lll}2 & 1 & 0\end{array}\right.$ & 12.8748 & 9.5963 & 0.0486 \\
$\left\{\begin{array}{lll}2 & 1 & 1\end{array}\right\}$ & 14.8665 & 12.1385 & 0.0443 \\
\hline
\end{tabular}


(a)

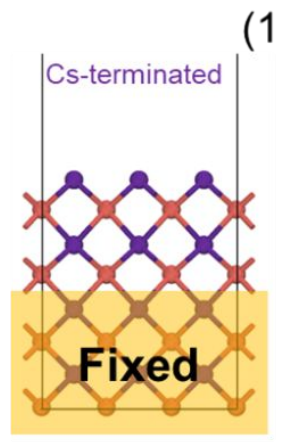

(d)

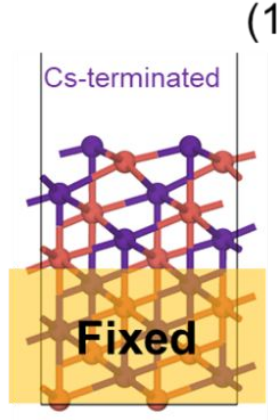

$\left(\begin{array}{lll}1 & 0 & 0\end{array}\right)$

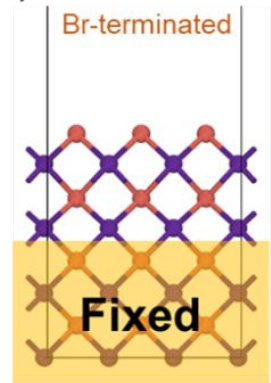

(111)

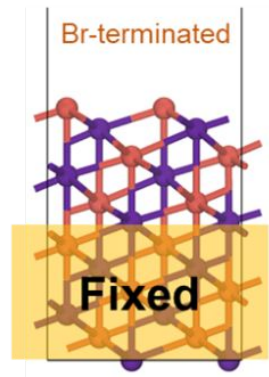

(b)

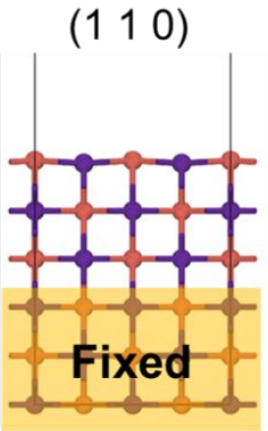

(c)

(2 11)

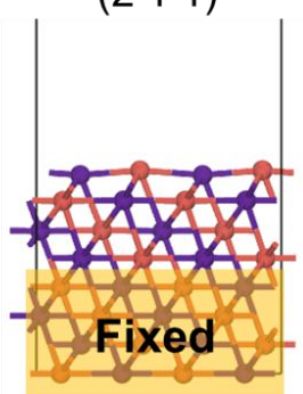

(e)

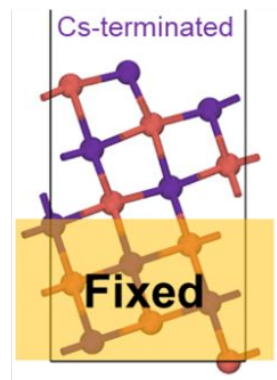

(2 10$)$

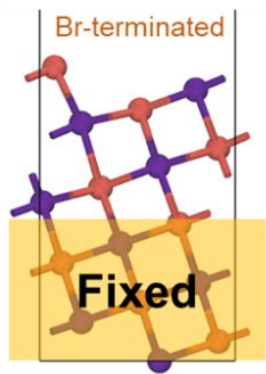

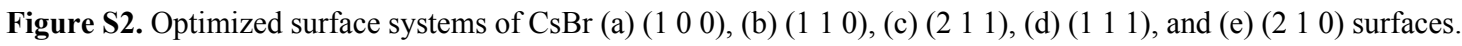
$\left(\begin{array}{ll}1 & 0\end{array}\right),\left(\begin{array}{lll}1 & 1 & 1\end{array}\right)$, and $\left(\begin{array}{lll}2 & 1 & 0\end{array}\right)$ surfaces are the polar surface which have two different kinds of termination: Cs- and Brtermination. $\left(\begin{array}{lll}1 & 1 & 0\end{array}\right)$ and $\left(\begin{array}{lll}2 & 1 & 1\end{array}\right)$ surfaces are the non-polar surface. $\mathrm{Cs}$ and $\mathrm{Br}$ are represented by purple and brown colored spheres, respectively. The bottom yellow regions were fixed to theoretically mimic the bulk phase.

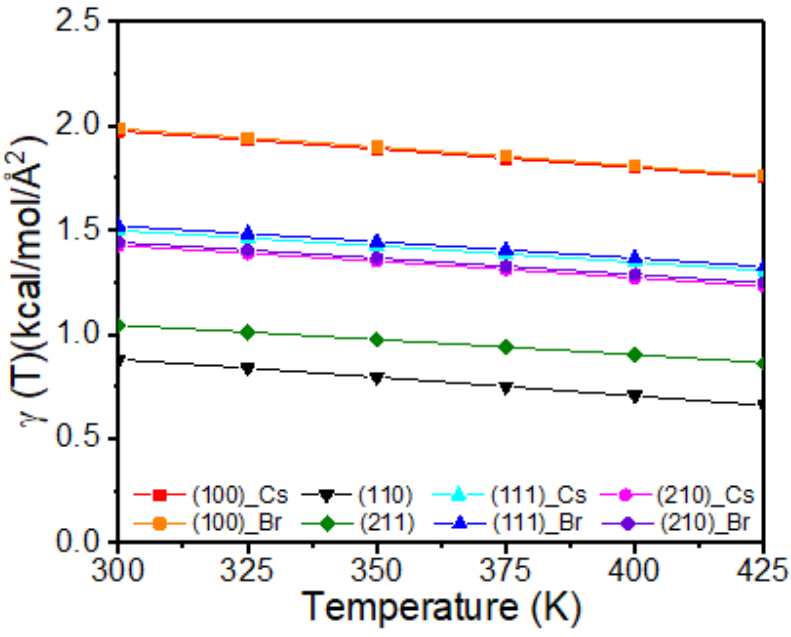

Figure S3. Surface free energy $(\gamma(\mathrm{T}))$ as a function of the temperature. 
(a)

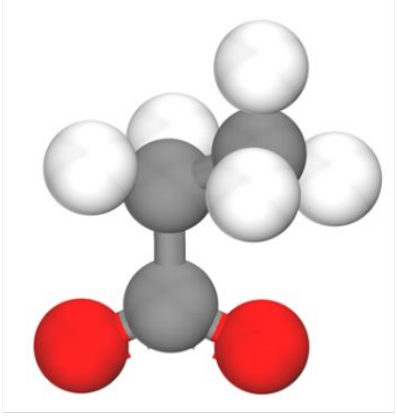

(b)

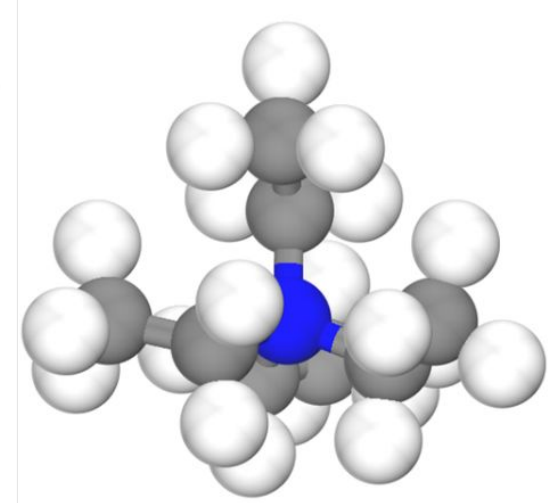

Figure S4. Optimized surfactant models; (a) oleate and (b) tetrabutylammonium (TBA). The propanoate ion and ethylammonium ion were used in place of oleate and TBA, respectively, to reduce the computational cost, as a simple model. $\mathrm{O}, \mathrm{N}, \mathrm{C}$, and $\mathrm{H}$ are represented by red, blue, grey, and white colored spheres, respectively. For the charge balance in adsorption free energy calculation of oleate and $\mathrm{TBA}, \mathrm{Cs}^{+}$and $\mathrm{Br}^{-}$are located in the vacuum region of surface system, respectively.

(a)

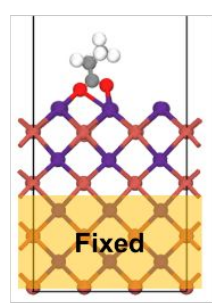

(1 111 )

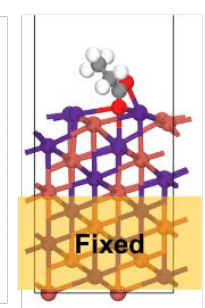

(2 110$)$

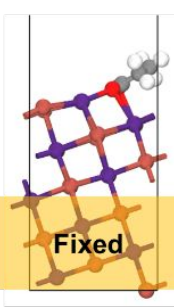

(11 10$)$

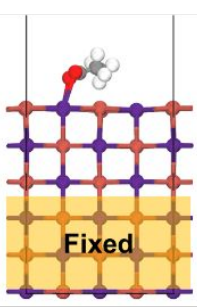

(2) 111$)$

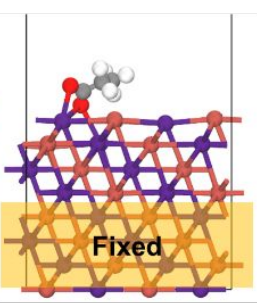

(b)

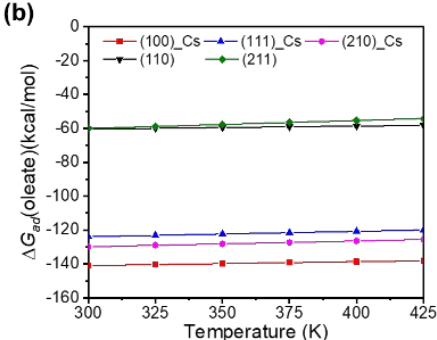

Figure S5. (a) Optimized structures of each $\mathrm{CsBr}$ surface with oleate model. For the polar surface, Cs-terminated surfaces are considered. The bottom yellow regions were fixed to theoretically mimic the bulk phase. $\mathrm{Cs}, \mathrm{Br}, \mathrm{O}, \mathrm{C}$, and $\mathrm{H}$ are represented by purple, brown, red, grey, and white colored spheres, respectively. (b) Adsorption free energies of oleate $\left(\Delta \mathrm{G}_{a d}\right.$ (oleate) $)$ as a function of the temperature.

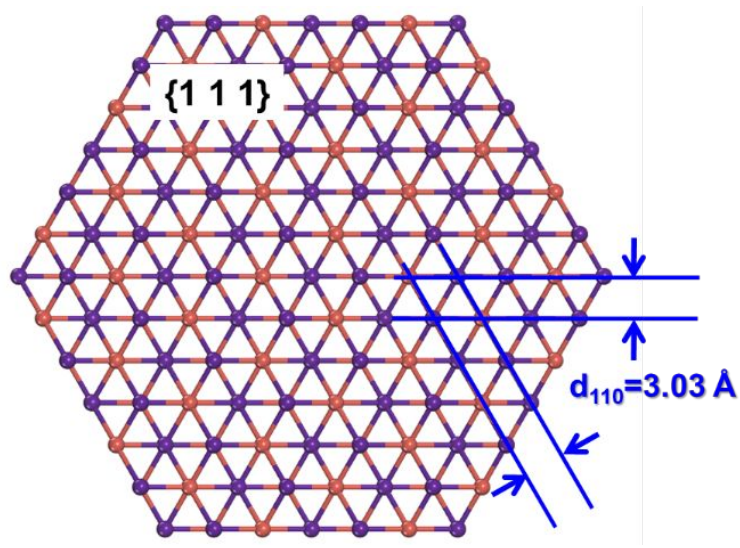

Figure S6. Projection view of schematic hexagonal model of hexagonal plate with d-spacing. Cs and $\mathrm{Br}$ are represented by purple and brown colored spheres, respectively. 

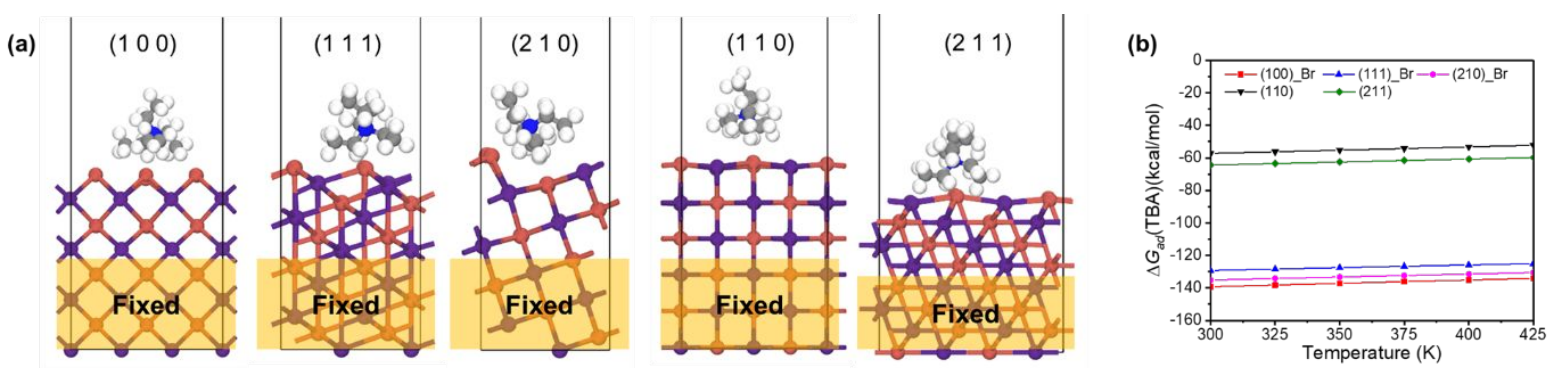

Figure S7. (a) Optimized structures of each $\mathrm{CsBr}$ surface with TBA model. For the polar surface, Br-terminated surfaces are considered. The bottom yellow regions were fixed to theoretically mimic the bulk phase. Cs, Br, N, C, and $\mathrm{H}$ are represented by purple, brown, blue, grey, and white colored spheres, respectively. (b) Adsorption free energies of TBA $\left(\Delta \mathrm{G}_{a d}(\mathrm{TBA})\right)$ as a function of the temperature.

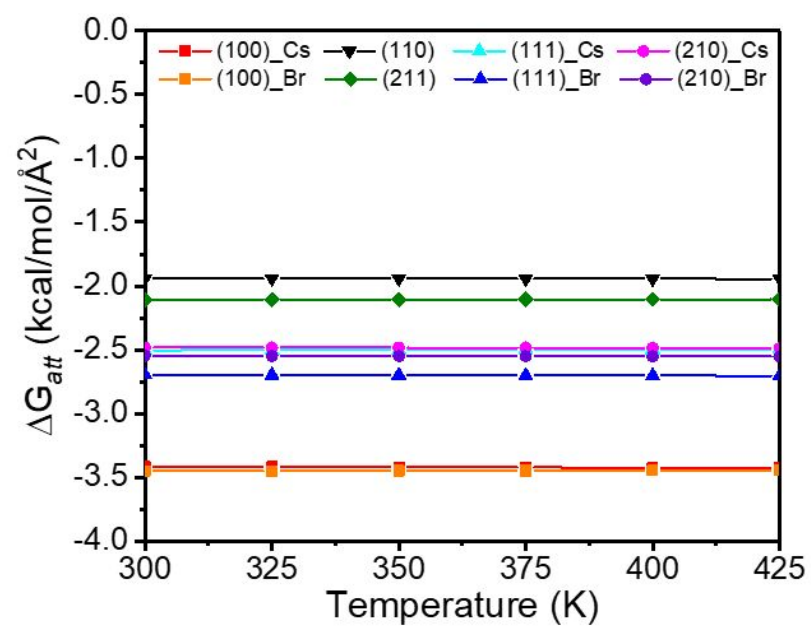

Figure S8. Attachment free energies $\left(\Delta \mathrm{G}_{a t t}\right)$ as a function of the temperature.

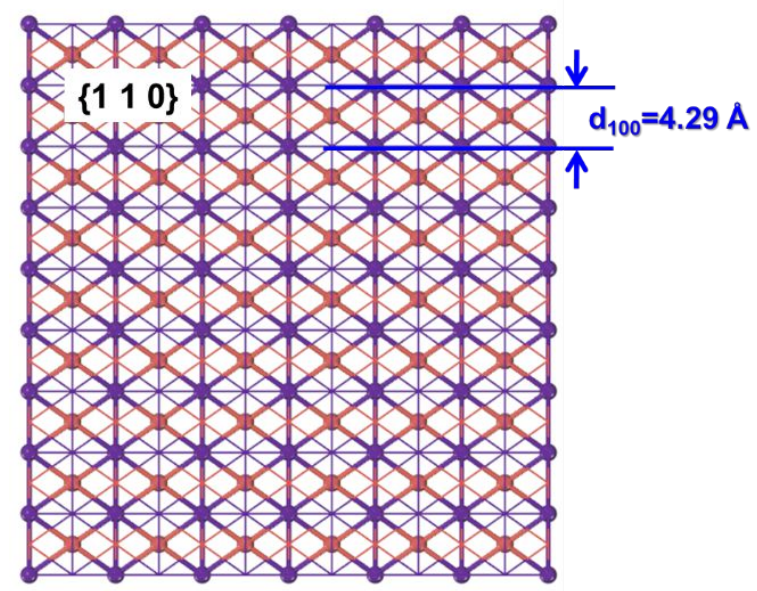

Figure S9. Projection view of schematic model of rectangular plate with d-spacing. Cs and $\mathrm{Br}$ are represented by purple and brown colored spheres, respectively. 


\section{REFERENCES}

(1) Rempel, J. Y.; Trout, B. L.; Bawendi, M. G.; Jensen, K. F. Density Functional Theory Study of Ligand Binding on CdSe (0001), (0001), and (1120) Single Crystal Relaxed and Reconstructed Surfaces: Implications for Nanocrystalline Growth. J. Phys. Chem. B 2006, 110 (36), 18007-18016.

(2) Liu, L.; Zhuang, Z.; Xie, T.; Wang, Y. G.; Li, J.; Peng, Q.; Li, Y. Shape control of CdSe nanocrystals with zinc blende structure. J. Am. Chem. Soc. 2009, 131(45), 16423-16429. 